Young-Gill Lee

Yoon-Jin $\mathrm{Ha}$

Seunng-Hee Lee

Sang Hyun Kim

http://dx.doi.org/10.21278/brod69105

ISSN 0007-215X

eISSN 1845-5859

\title{
A STUDY ON THE ESTIMATION METHOD OF THE FORM FACTOR FOR A FULL-SCALE SHIP
}

UDC 629.5.015.2:629.5.016.7:629.5.018.72:519.6

Original scientific paper

\begin{abstract}
Summary
In this study, a prediction method of the form factor for a full-scale ship is suggested to minimize the power prediction error from a small model ship. Numerical simulations were carried out at various Reynolds numbers from a small model to a full-scale ship. The variation of the form factors was investigated from the results of the numerical simulation according to the Reynolds numbers. In addition, the results from the numerical simulations and experimental data of the geosim models were utilized to drive the correlation line and predict the form factor of a full-scale ship. The correlation line was applied to predict the effective power and the delivered power of a full-scale ship. As a result, the developed prediction method confirmed the possibility of predicting the power reliably from experiments using a small model.
\end{abstract}

Key words: $\quad$ Reynolds number; Form factor; Effective power; Delivered power; Geosim models

\section{Introduction}

Model tests in towing tanks are indispensable for predicting the resistance or the effective power and delivered power of a full-scale ship. In the model tests, the models are towed at speeds scaled to coincide with the Froude numbers of a full-scale ship at the given speeds. Therefore, the Reynolds numbers of the model ship and full-scale ship are different; hence, the frictional resistance and form factor are different. Van et al. (2011) reported that the residual resistance coefficients decrease as the size of the model ship increases, whereas form factors become larger.

The pressure coefficients recovery for the after part becomes faster as the Reynolds number is increased. As a result, the residual resistance coefficient decreases. And Choi et al. (2011), García-Gómez (2000) and Min and Kang (2010) also reported that the form factors increase with increasing Reynolds number.

The effective wake fraction is also decreased because the axial velocity on the propeller plane increases with increasing Reynolds number. All the results suggest that use of larger 
models is advantageous for reliable prediction of the effective power or delivered power of a full-scale ship. In a relative small towing tank, however, the size of the model is limited $\left(\operatorname{Re}=10^{6} \sim 10^{7}\right)$ and a reliable prediction of the effective power or delivered power of a fullscale ship through model tests are not always easy.

Moreover, the scale ratios of a model ship and full-scale ship increase with decreasing size of a ship. In addition, model tests are performed at low Reynolds numbers because the speed of a ship decreases with a model ship size by the Froude scaling. In other words, reasonable prediction of the effective power and delivered power are difficult due to the scale effects.

A low-speed blunt ship gradually appears by the down speeding and size-up of a ship. Generally, the low-speed blunt model ship is large with an intense bilge vortex for the after part. The bilge vortex is caused by the flow field of the hook shape that is separated from bilge of a ship. The bilge vortex is related to the thickness of the boundary layer.

In the ITTC 1978 method, the form factors are kept constant and show small differences in the Reynolds numbers, inevitably showing a difference between the model and full-scale ship. Min and Kang (2010) proposed a method to remedy these problems using only the results of model tests in the range of $\operatorname{Re}=10^{6} \sim 10^{7}$. They adopted a hyperbolic function defined as the ratio between the form factors of a full-scale ship and the model and the Reynolds numbers. On the other hand, the method did not take the differences in the hull forms into consideration and the predicted powers for high speed ships, such as KCS are prone to substantial error. The procedure reported by Ha et al. (2013) is similar to Min and Kang (2010) method except that they adopted an exponential function. This method, however, was devised to take the differences in hull forms among low, middle and high speed ships into consideration, resulting in better prediction for high speed hull forms than those obtained using Min and Kang's method. In the present study, KVLCC2, KLNG and KCS were selected as the typical hull forms of low, middle and high-speed vessels, respectively. To estimate the dependency of the form factor on the Reynolds number, the form factors of three hull forms were computed numerically at several Reynolds numbers corresponding to the model and the full-scale ship. The form factors found from a numerical computation were combined with those from the tests of the geosim model and went through regression analysis to construct rational correlation lines for model-ship extrapolation. The lines are applied to the power prediction of the target ships and yield marked improvement.

\section{Reference Hull Form}

The principal dimensions of the ships, KVLCC2, KLNG and KCS are open to the public and can be found in Ha et al. (2013) or elsewhere. Table 1 shows lists the design speeds of the ships and various scale ratios taken for the model tests and numerical simulations.

Table 1 Design speeds and scale ratios of the ships; KVLCC2, KLNG and KCS

\begin{tabular}{|c|c|c|c|}
\hline Ship name & KVLCC2 & KLNG & $\mathrm{KCS}$ \\
\hline Design speed & $15.5 \mathrm{Knot}$ & $19.5 \mathrm{Knot}$ & 24.0Knots \\
\hline $\begin{array}{l}\text { Scale ratios for } \\
\text { model tests }\end{array}$ & $1 / 160,1 / 100,1 / 58$ & $1 / 103,1 / 69,1 / 34$ & $\begin{array}{c}1 / 125,1 / 97,1 / 63.2 \\
1 / 31.6\end{array}$ \\
\hline $\begin{array}{l}\text { Scale ratios for } \\
\text { numerical } \\
\text { simulations }\end{array}$ & $\begin{array}{c}\text { 1/160, 1/100, 1/58, } \\
1 / 29,1 / 10,1 / 5 \\
\text { full-scale }\end{array}$ & $\begin{array}{l}1 / 103,1 / 69,1 / 34, \\
1 / 10,1 / 5, \text { full-scale }\end{array}$ & $\begin{array}{c}1 / 160,1 / 97,1 / 63.2 \\
1 / 31.6,1 / 21,1 / 10 \\
1 / 5, \text { full-scale }\end{array}$ \\
\hline
\end{tabular}




\section{Power Prediction Method for A Full-Scale Ship}

Three-dimensional extrapolation method recommended by ITTC are generally used to predict the effective power of a full-scale ship by a model test. Fig. 1 explains the power prediction of a full-scale ship. This method is based on Froude's law and the wave-making resistance coefficient and form factor of a full-scale ship, and its model ship are assumed to be the same.

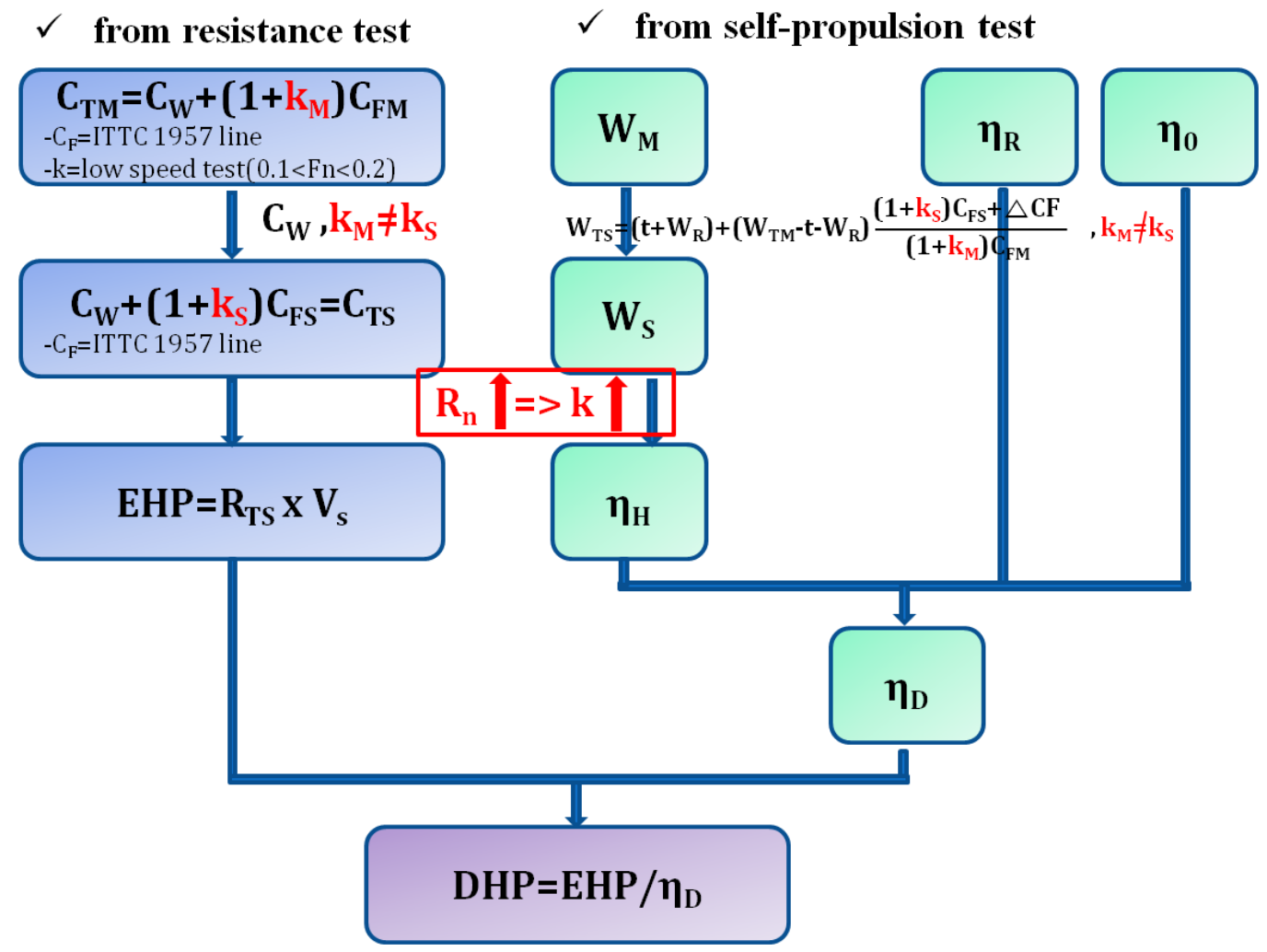

Fig. 1 Power prediction of a full-scale ship by ITTC

Fig. 2 compares the pressure contours for the model ships and full-scale ships on the side profile of the reference hull forms by the numerical simulations. The numerical simulation conditions are explained at validation part of the numerical simulation in this paper. The pressure contours of the reference hull forms are similar for the front parts of the model ships and full-scale ships. On the other hand, the positive pressure contours near the sterns of the reference hull forms grow on the full-scale ship. Consequently, the pressure coefficient of the full-scale ship is smaller than the pressure coefficient of the model ship. In other words, the residual resistance coefficients increase with decreasing Reynolds number. Ha et al. (2012) and Van et al. (2011) clearly showed that the residual resistance coefficients increase with decreasing model ship size according to the geosim model about the reference hull forms tests. Therefore, to predict the effective power of a full-scale ship by a model test, two-dimensional extrapolation methods recommended by ITTC is difficult from the small model ship. The two-dimensional extrapolation method is based on Froude's law, and the residual resistance coefficient of a full-scale ship and its model ship are assumed to be the same. On the other hand, the wave resistance coefficients of a full-scale ship and model ship are similar because the Froude numbers of a full-scale ship and model ship are the same. As shown in Fig.3, the wave profiles of a full-scale ship and model ship of the Series $60 \mathrm{CB}=0.6$ by the numerical calculation are similar. Therefore, the wave resistance coefficient is the dominant value by the Froude number. 


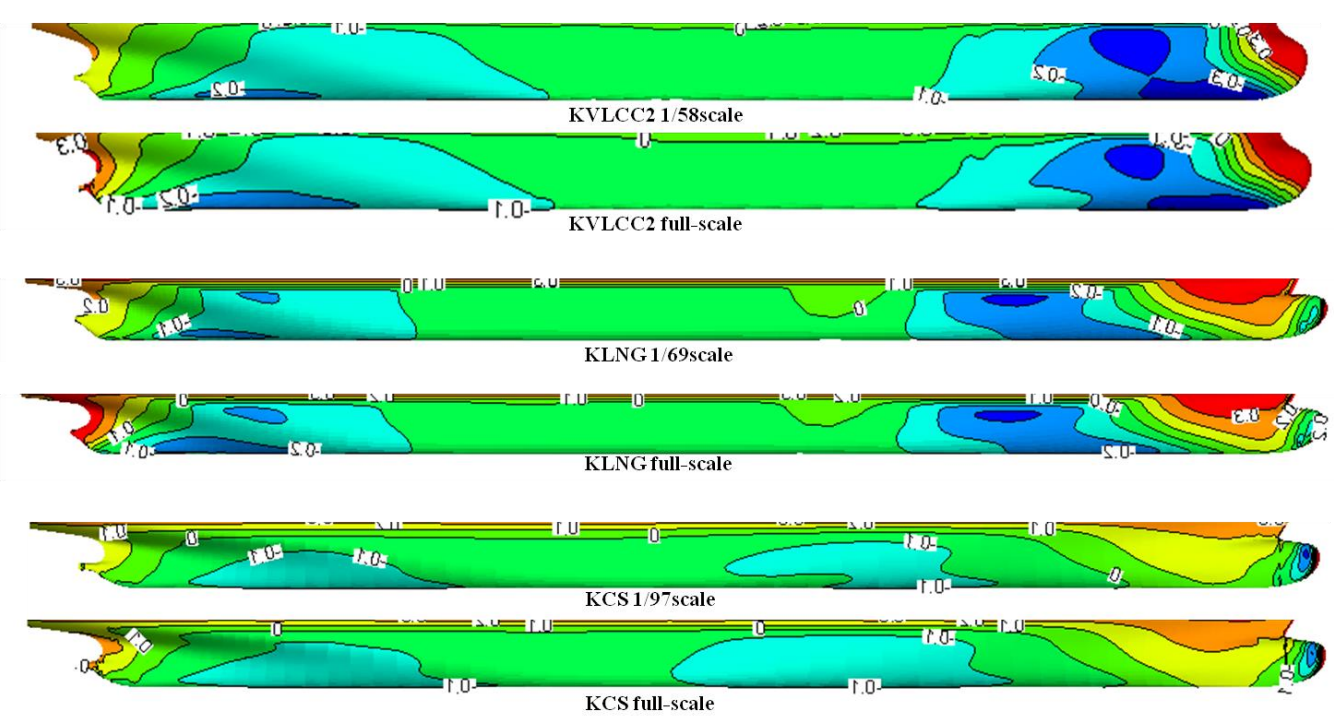

Fig. 2 Comparisons of the pressure contours on the hull surfaces

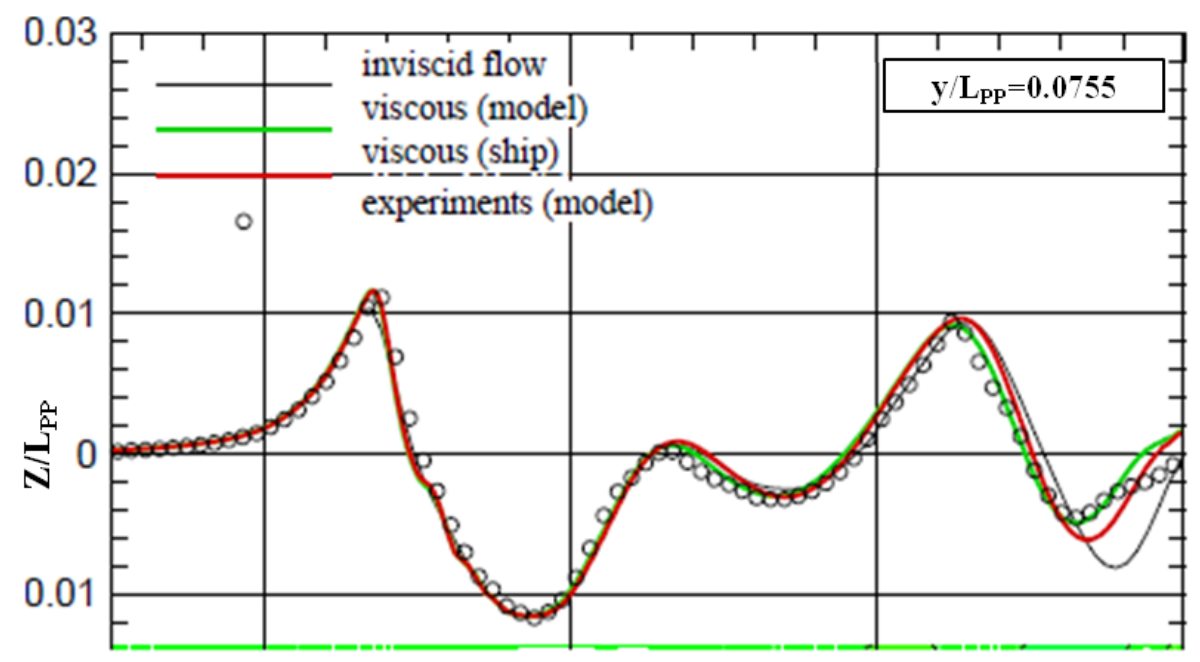

Fig. 3 Wave profile of the Series $60 C_{B}=0.6$ (Raven et al., 2010)

Therefore, the three-dimensional method (ITTC 1978 method) takes the Reynolds number dependency of the resistance components into account in the extrapolating procedure by introducing the invariant form factors, which are generally believed to yield better results, and were used in this study. On the other hand, the form factor is a function of the Reynolds number and the values for a full-scale ship and the model should be different.

Therefore, to predict the effective power of a full-scale ship, the ITTC 1978 method needs to improve because the ITTC 1978 method does not consider the difference of the form factor of the full-scale ship and model ship. As shown in Fig. 1, the prediction of the effective wake of a full-scale ship is related to the form factor, so the predicted delivered power of a full-scale ship is also needed. Fig. 4 shows the axial velocity contours on the propeller plane of the reference hull forms according to the Reynolds numbers by the model test. where, The IUTT is model test results in Inha university towing tank and other model tests results is reference data by Yang et al.(2010) and Van et al. (2011). The axial velocity accelerated notably with increasing model size and the hook shape in the axial velocity contour decreased with increasing model size. Hence, the prediction result of the effective wake of a ship and the 
prediction result of a nominal wake of a ship are different because the velocity contours on the propeller plane differ according to the model ship size. Therefore, the prediction delivered power of a full-scale ship needs to consider the difference of the form factors of a model ship and full-scale ship.
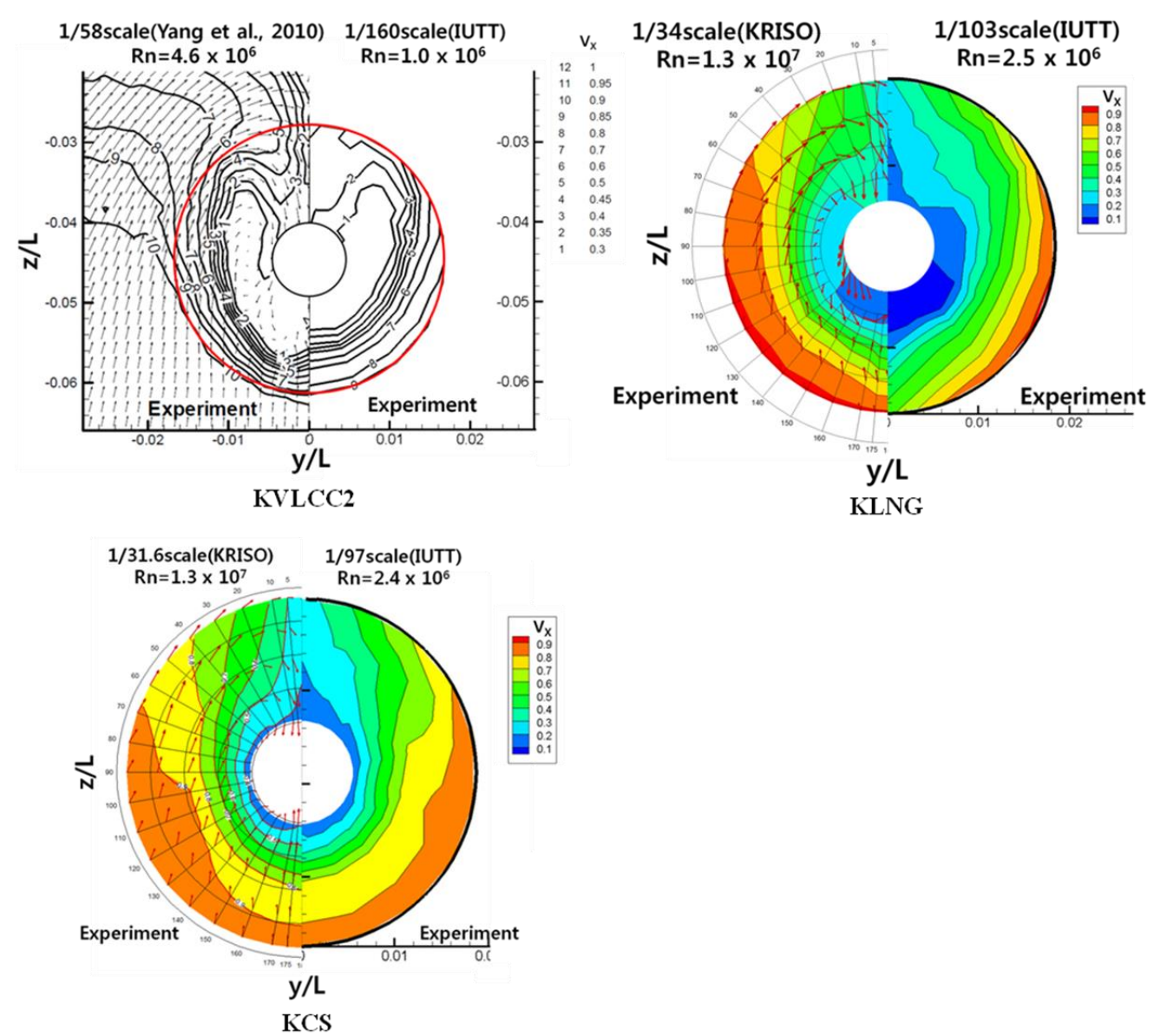

Fig. 4 Axial velocity contours on the propeller plane of KVLCC2, KLNG and KCS according to the Reynolds numbers

García-Gómez (2000) and Min and Kang (2010) examined the Reynolds number dependency of the form factor and proposed methods to estimate the form factor at a fullscale from the results of model experiments. Ha et al. (2013) also estimated the total resistance coefficient of the reference hull forms in the model scales using the two methods. The method suggested by García-Gómez (2000), however, yielded larger discrepancies in the predicted total resistance coefficients if the model sizes were altered because a change in the form factor due to the variation of the Reynolds number was not considered sufficiently. The method suggested by Min and Kang (2010) produced a similar prediction of the effective power of a full-scale ship to the method suggested by García-Gómez (2000). The predicted effective powers of the full-scale ship were still different. Because the method suggested by Min and Kang (2010) did not consider the difference according to the hull forms, the model test results by Min and Kang (2010) showed considerable scattering of the form factors. Therefore, the reasonable prediction method for the effective power and delivered power of a full-scale ship is needed; hence, a study of the precise prediction form factor of a full-scale ship is needed. 


\section{New Power Prediction Method for A Full-Scale Ship}

\subsection{Validation of the Numerical Simulation}

In this study, the numerical simulations were performed using commercial code, FLUENT. The governing equations are the continuity equation and Navier-Stokes equations. Navier-Stokes equations were solved by SIMPLE (Semi-implicit Method for Pressure Linked Equation). The second order upwind difference method was used for spatial discretization in convection term. The VOF (Volume of Fluid) method was applied to define the location of the free surface. When careful examinations of the grid generation and decision of the turbulence model are needed, the numerical simulations at high Reynolds numbers are very important for deriving similar numerical simulation results to the real flow phenomena. In the case of a numerical calculation of a full-scale ship, the numerical simulation results are compared with the model ship test results because of the difficult obtainment of sea trial data and measurement data of a full-scale ship. Fig. 5 shows the typical grid system regarding KVLCC2 for the numerical simulation, and Table 2 lists the number of grids and the size of $\mathrm{y}+$.

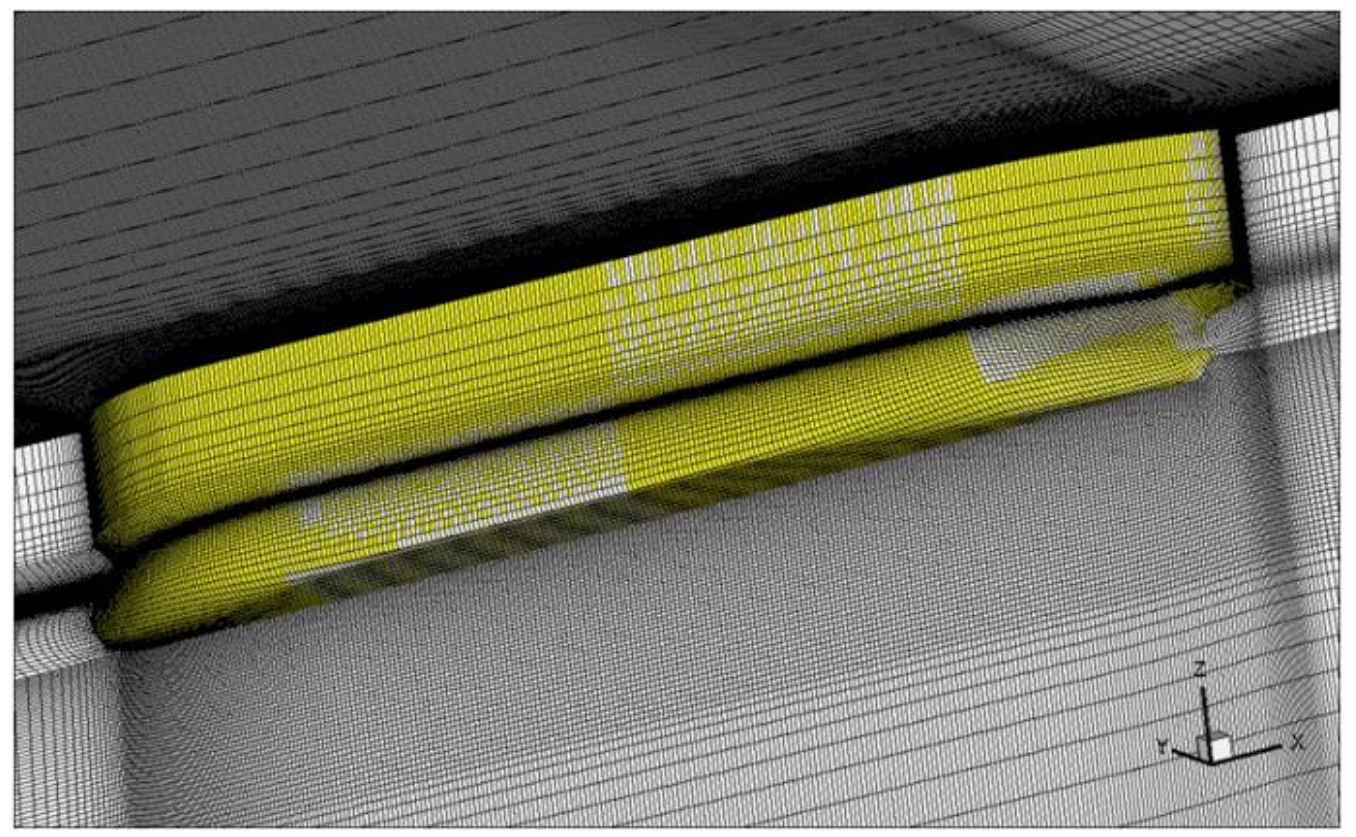

Fig. 5 Grid system of KVLCC2

Table 2 Grid information of the numerical simluation

\begin{tabular}{|c|c|c|}
\hline & Full-scale & Model \\
\hline Number of cells & \multicolumn{2}{|c|}{$1.6 \times 10^{5}$} \\
\hline $\mathrm{y}+$ & 900 & 40 \\
\hline
\end{tabular}

The number of grids of a full-scale ship was obtained from Choi and Kim (2010). In the study by Choi and Kim (2010), the numerical simulations regarding the pipe, flat plate and axis of symmetry were performed to survey the used $y+$, and the change in the numerical values were studied according to the increase in y+. In addition, Choi and Kim (2010) suggested an equation for calculating $\mathrm{y}+$ above $\mathrm{Re}=10^{7}$. In this study, the equation suggested 
by Choi and Kim (2010) was used to derive y+ for the numerical simulation of a full-scale ship. The numerical simulations for validation regarding the turbulence model in this study were performed on a KVLCC2 $1 / 58$ scale model because the KVLCC2 is dominatedant by the viscous effect and the design speed of the KVLCC2 is the slowest of the reference hull forms. In addition, the numerical simulation results were compared with the model test results by the KRISO (Korea Research Institute of Ships \& Ocean Engineering). First of all, the flow fields of the numerical simulation results for decision of the proper turbulence model were compared with the results reported by Kim and Kim (2006) and Yang et al. (2010). In these numerical simulations, the turbulence models used were the Realizable k- $\varepsilon$ and Reynolds stress models. The reasonable numerical simulations of the flow field of the after part as well as the free surface were performed because the numerical simulations in this study contained the free surface. On the other hand, the numerical simulation results reported by Kim and Kim (2006) and Yang et al. (2010) were both obtained using the double model.
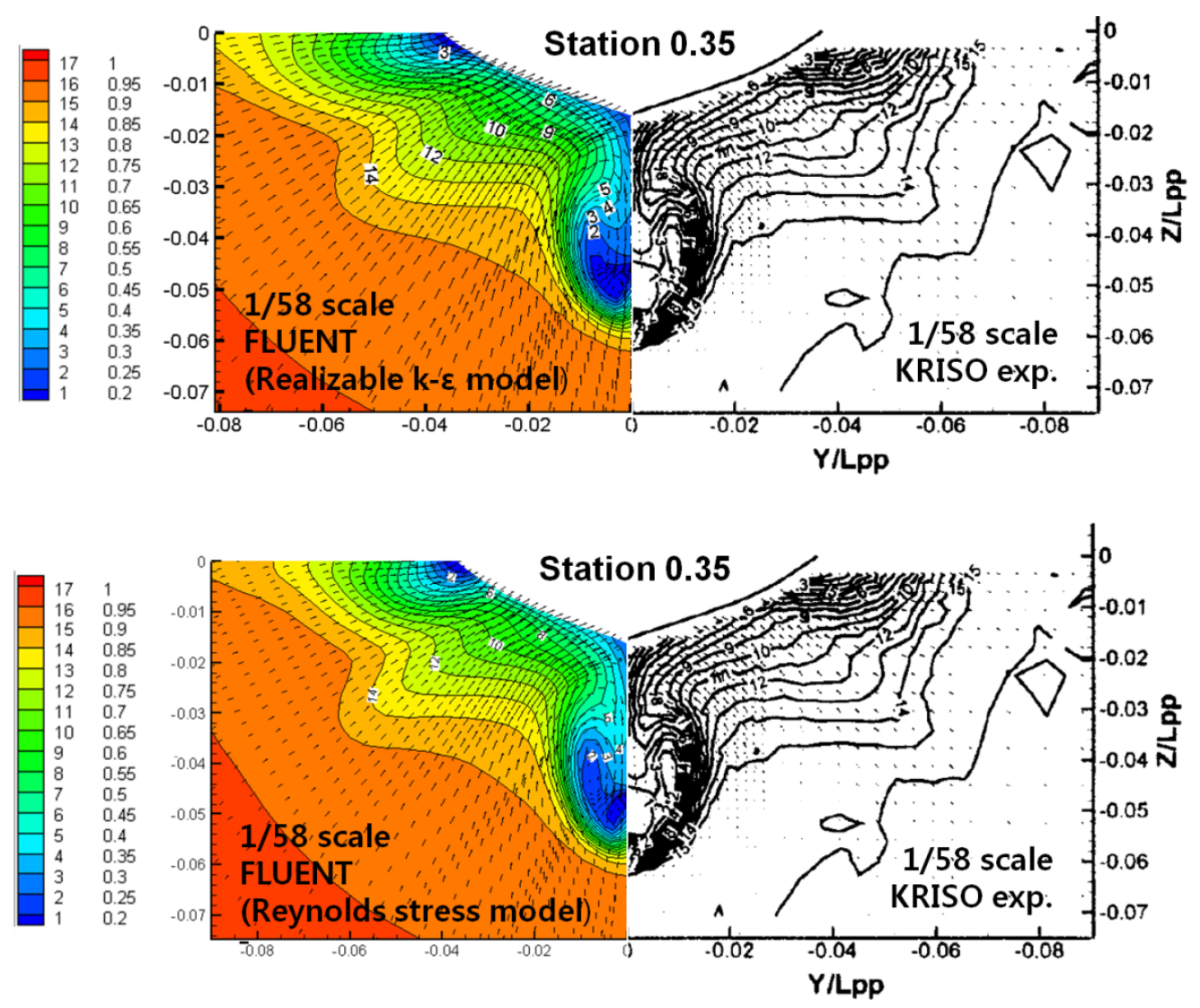

Fig. 6 Comparison of the wake field using the Realizable k- $\varepsilon$ and Reynolds stress models

Therefore, these numerical simulation results did not contain the free surface. The numerical results by the Reynolds stress model showed similar model test results to the KRISO reported by Kim and Kim (2006) and Yang et al. (2010). Fig. 6 shows the numerical simulation results by the Realizable k- $\varepsilon$ and Reynolds stress models. In addition, similar to the studies reported by Kim and Kim (2006) and Yang et al. (2010), the numerical simulation results by the Reynolds stress model with the free surface showed similar model test results to the KRISO in this study. It is well-known that the Reynolds stress model has a degree of high order for the Realizable k- $\varepsilon$ model. Therefore, it can be found that the size of hook shape by Reynolds stress model was relatively larger than the results by the Realizable k- $\varepsilon$ model. Fig. 
7 shows the wave contours and wave height on the free surface. The numerical simulation results of the wave contours in this study were slightly high for the model test results of the wave contours by KRISO, but these results were qualitatively similar. In addition, in the wave height, the numerical simulation results of the wave height was quantitatively similar to the model test results by KRISO. Therefore, the numerical simulation schemes in the present study are appropriate in the numerical simulation for the free surface, and the numerical simulation schemes in this study could be similarly predicted using the model test results.

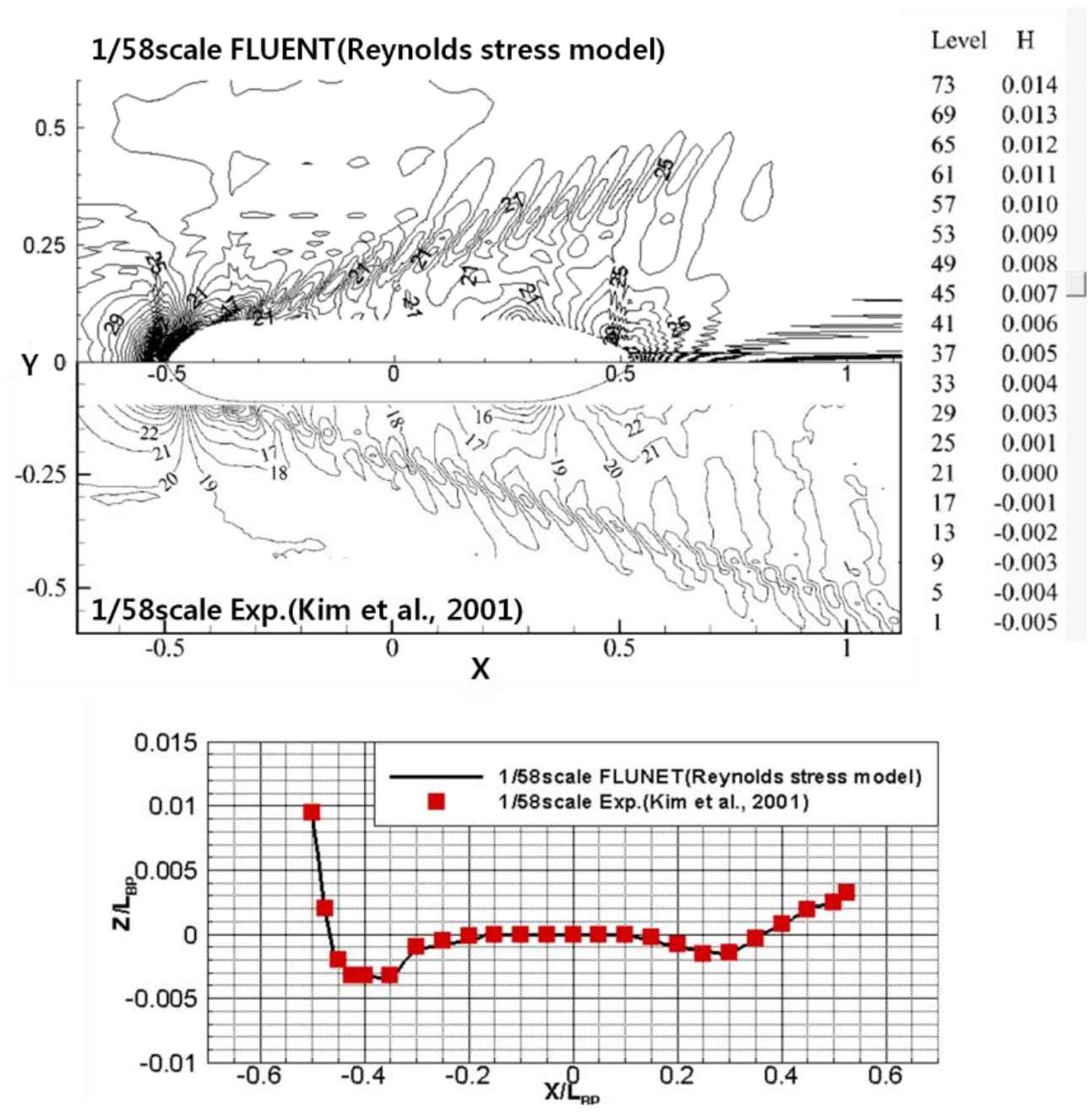

Fig. 7 Comparisons of the free-surface wave contours and wave height

\subsection{Power Prediction Method for a Full-Scale Ship}

In this study, numerical simulations of the ship resistance were performed to examine the influence of the Reynolds number on the form factor. The estimated form factors from the numerical simulations were added to those from the model tests to suggest a rational prediction method for predicting the form factor on a full-scale because the model tests were performed at a low Reynolds number, typically in the range of $\operatorname{Re}=10^{6}-10^{7}$. Therefore, in the model test for the transient region, the model tests could be reasonably performed by inducing the turbulence flows, because laminar and turbulence flows coexist. Generally, studs are used for turbulence stimulations. In this study, however, turbulence stimulators, such as studs and wires were installed near the 19.5 station to ensure reliable model test results. As recommended by ITTC, the form factor is determined using Prohaska's method, which 
assumes the form factors are a linear function a function of $\mathrm{Fn}^{4}$. Fig. 8 compares the estimated form factors, plotted as a function of the model length, by model test and numerical simulation for the three test ships.

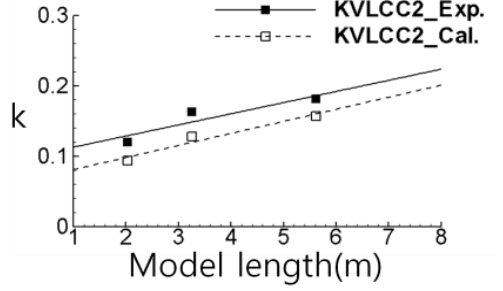

(a) KVLCC2

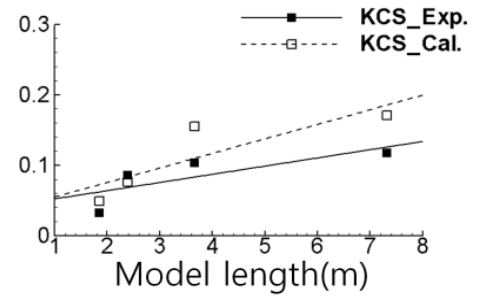

(c) KCS

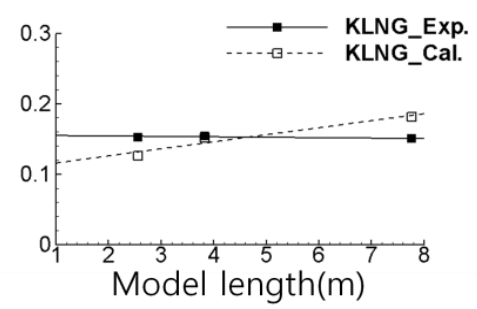

(b) KLNG

Fig. 8 Comparisons of the form factors by the model test and numerical simulation

Fig. 8 shows that the estimated form factor of the numerical simulations are generally in good agreement with those found from the model tests. In addition, both estimated form factors increase with increasing model length. Fig 9 shows the non-dimensional x-velocity contours at each stations about the $2 \mathrm{~m}$ class model ships and full-scale ships of the reference hull forms, and these non-dimensional x-velocity contours are the results below the boundary layers. In the non-dimensional x-velocity contours about the model ships of the reference hull forms, the bilge vortex appeared from near the 1 station around KVLCC2 and KLNG of the middle-low speed vessels. But the bilge vortex was barely detectable in KCS of the high speed vessels. In non-dimensional $x$-velocity contours about the full-scale ships of the reference hull forms, the bilge vortex was barely observable for the model ships. On the other hand, a low speed region of the middle-low speed vessels such as KVLCC2 and KLNG appeared for KCS in the case of the full-scale ships. As shown in Fig. 9, the stern lengths of the KVLCC2 and KLNG area were shorter than the stern length of the KCS, and the water plane shapes at the loaded water line were different, respectively. In other words, the appearance locations of the bilge vortex were similar in the middle-low speed vessels such as KVLCC and KLNG model ships, but the bilge vortex was barely observable around the KCS model. The flow phenomena around the KVLCC2 and KLNG ships were similar but the phenomena around the KCS ship was different. Therefore, the form factors as a function of the Reynolds number of the KVLCC 2 and KLNG could be similar, and the rising trend of the form factors could be both similar as the Reynolds number increases. The form factors of the KCS could be relatively lower than the form factors of the middle-low speed vessels, and the increasing trend of the form factors of the KCS could be different for the middle-low speed vessels. The study by Min and Kang (2010) was suggested to estimate the form factor of a full-scale ship, but this study did not consider the differences in the hull forms. On the other hand, the middle- low speed ships and high speed ships were classified according to the differences in the hull forms in this study. 
Young-Gill Lee, Yoon-Jin Ha

Seunng-Hee Lee, Sang Hyun Kim
A Study on the Estimation Method of the form factor for a full-scale ship

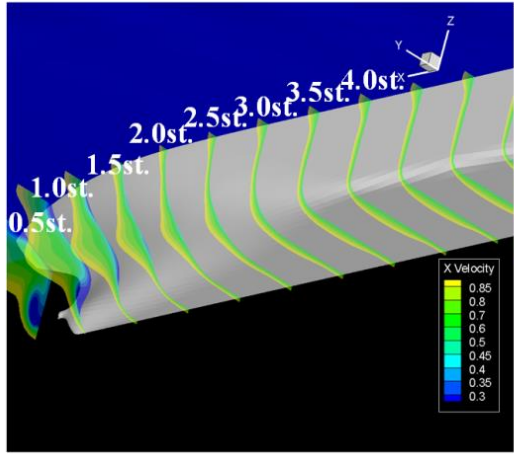

KVLCC2 1/160scale

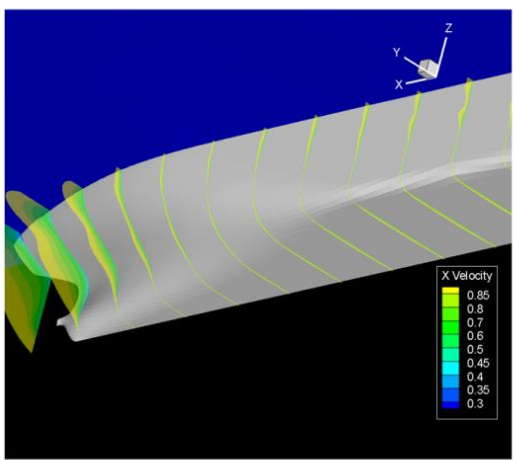

KVLCC2 full-scale

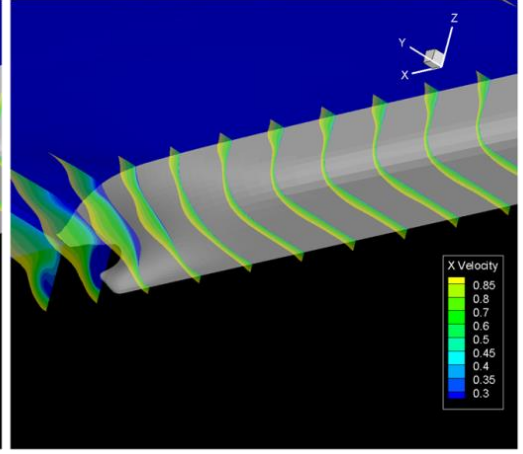

KLNG 1/103scale

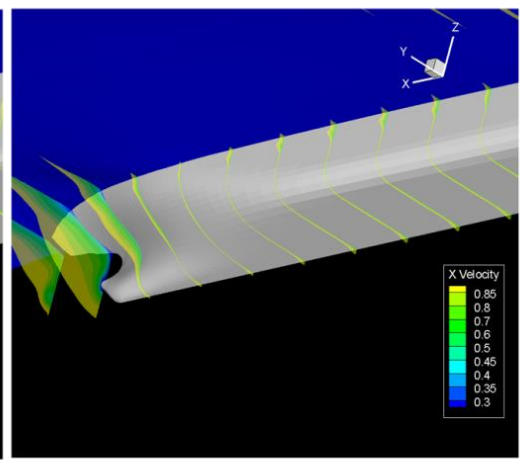

KLNG full-scale

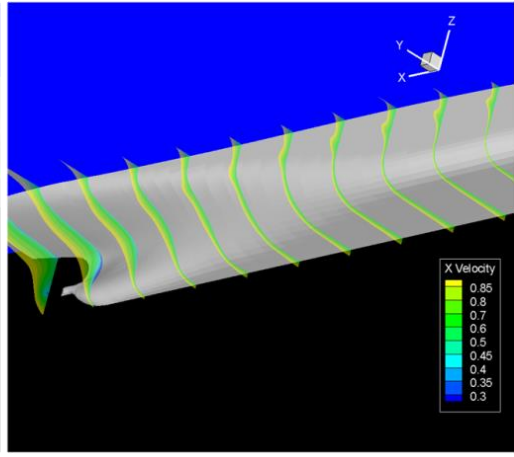

KCS 1/97scale

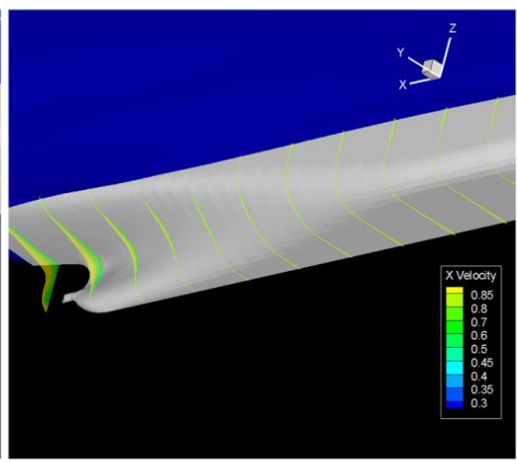

KCS full-scale

Fig. 9 Comparisons of the $x$-velocity according to the station

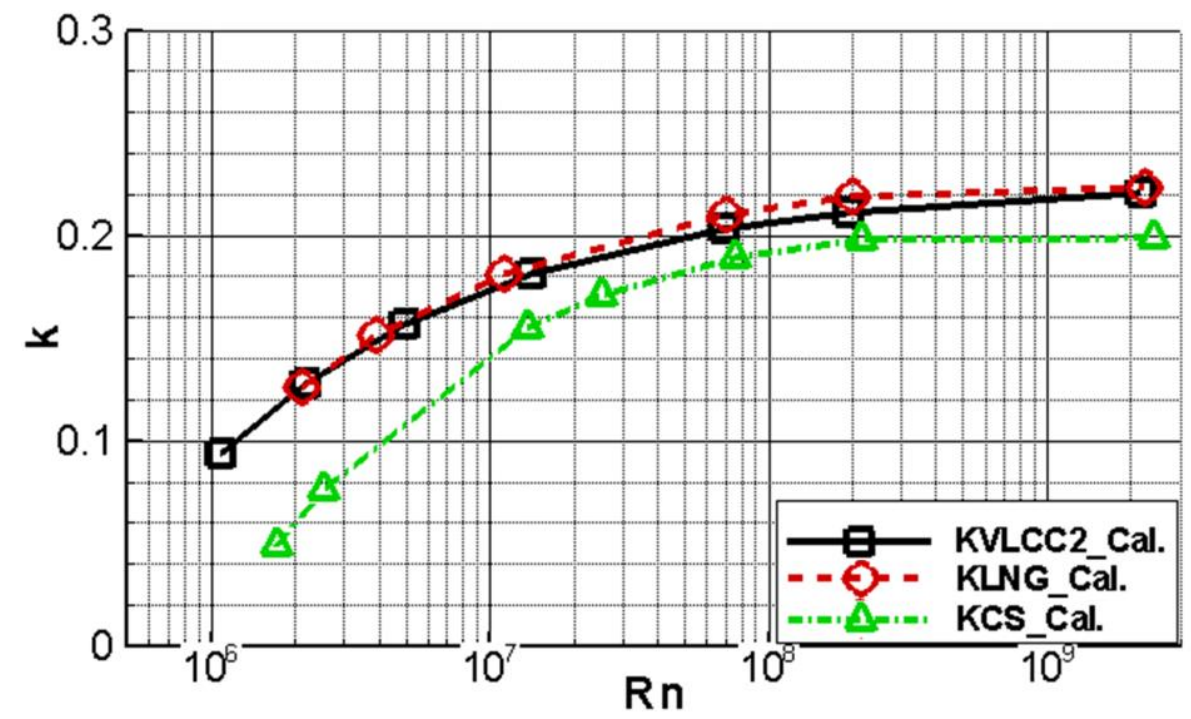

Fig. 10 Variation of the numerically estimated form factors with the Reynolds number

Fig. 10 shows the variation of the form factors estimated by the numerical computations with the Reynolds number. The form factors increase rapidly with increasing Reynolds number in the range of $\mathrm{Re}=10^{6} \sim 10^{7}$. Moreover, the tendency of the estimated form factors for KVLCC2 and KLNG almost coincide with each other but that of KCS is separate from the other. Therefore, the tendency of form factors for middle-low speed ships is quite different with high speed ships. The form factor increases with increasing Reynolds number for the entire simulation range. Furthermore, as the Reynolds number approaches the full-scale value, 
the estimated form factor appears to converge to certain values. Accordingly, when predicting the effective power and delivered power of full-scale ship from the model test in $\operatorname{Re}=10^{6} \sim 10^{7}$, the variation of the form factor with the Reynolds number should be considered.

Fig. 11 shows the model test and numerical simulation results by this study along with the model test results and the form factors estimation band of the full-scale ships reported by Min and Kang (2010) for validation of the numerical simulation results of the full-scale ships. The numerical simulation results of the full-scale ships by this study were contained in the form factors estimation band of the full-scale ships reported by Min and Kang (2010). Therefore, the numerical simulation results of the full-scale ships by this study could be used to improve the estimation method of a ship form factor. Min and Kang (2010) assumed that the form factor increases with increasing Reynolds number, and this form factor could converge a constant value to above the $\mathrm{Re}=10^{9}$, and is called the terminal form factor. The form factors converged to a constant value as the Reynolds number increases, shown in Fig. 10 and 11. Min and Kang (2010) performed regression analysis using a relative value, i.e., the ratio of the form factor with respect to the terminal form factor, and this the regression analysis result was called the form factor correction factor $\left((1+\mathrm{k})_{\mathrm{M}} /(1+\mathrm{k})_{\infty}\right)$. The form factor correction factor converged as the Reynolds number increased, and was 1.0 when the Reynolds number was greater than $10^{9}$. Ha et al. (2013) referred to the concepts by Min and Kang (2010).

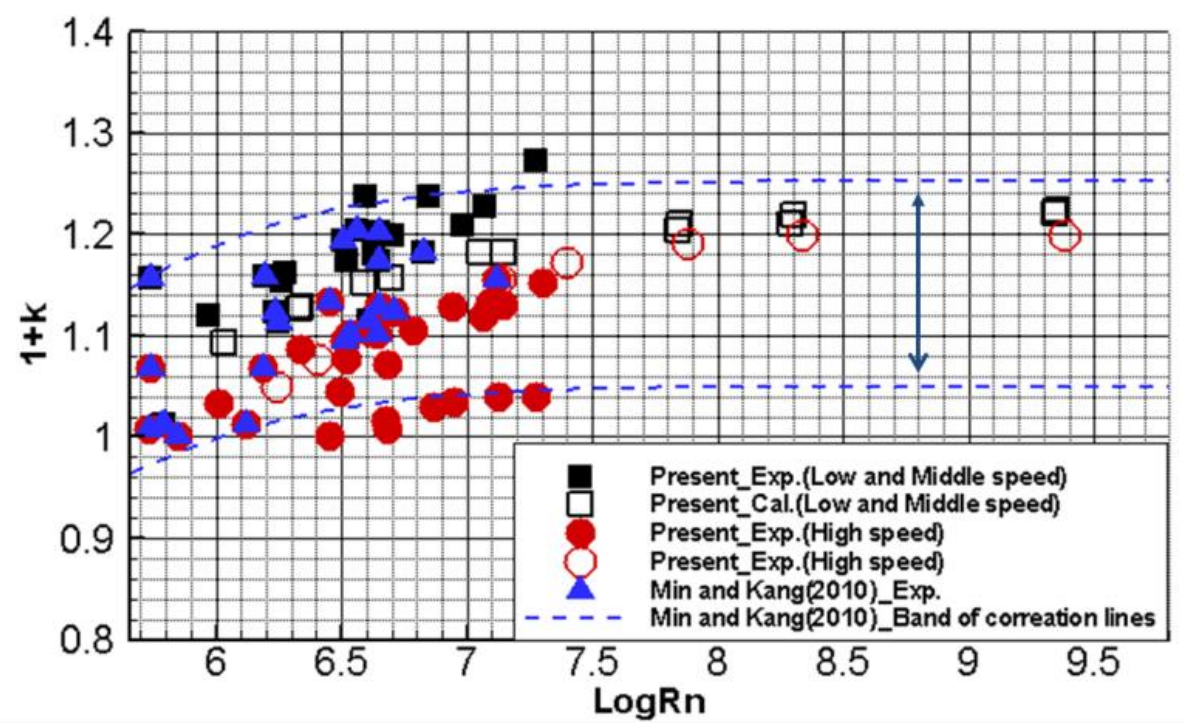

Fig. 11 Comparisons of the form factors with the Reynolds number

Fig. 12 compares correlation lines of the form factors by Min and Kang (2010) and Ha et al. (2013). The correlation line of Min and Kang (2010) was applied as a hyperbolic function, and the correlation line of Ha et al. (2013) was applied as an exponential function. Unlike the correlation line of Min and Kang (2010), the correlation line of Ha et al. (2013) can be classified into two lines. On the other hand, the form factor of a full-scale ship is difficult to estimate from all of the correlation lines because the model test data are nonexistent in the region of $\mathrm{Re}=10^{8} \sim 10^{9}$ (region of a full-scale ship). In this study, the numerical simulation data in Fig. 10 and 11 were used in regression analysis. Regression analyses for an improvement of the method reported by Ha et al. (2013) were performed according to the assumption and concept by Min and Kang (2013) and the correlation line of form factors by Ha et al. (2013). 


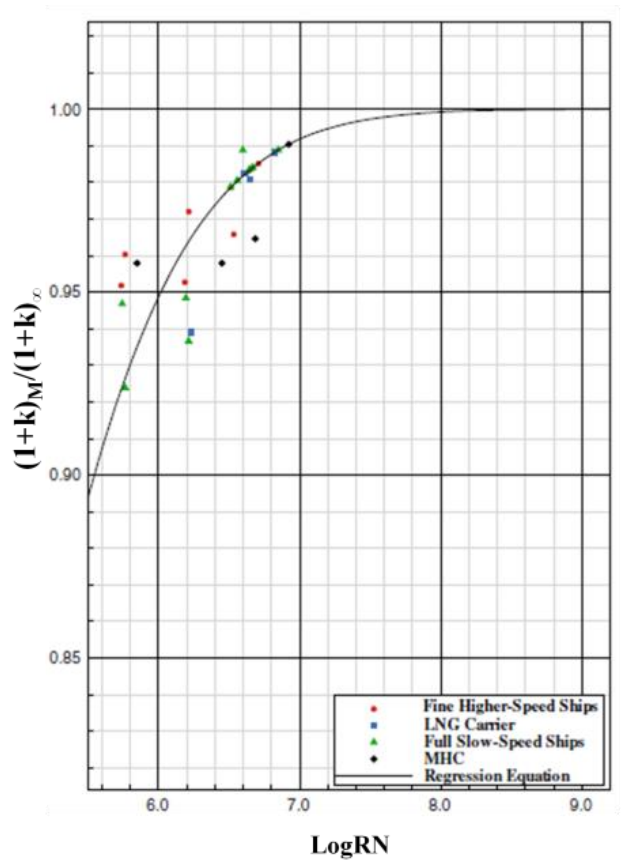

Min and Kang(2010)

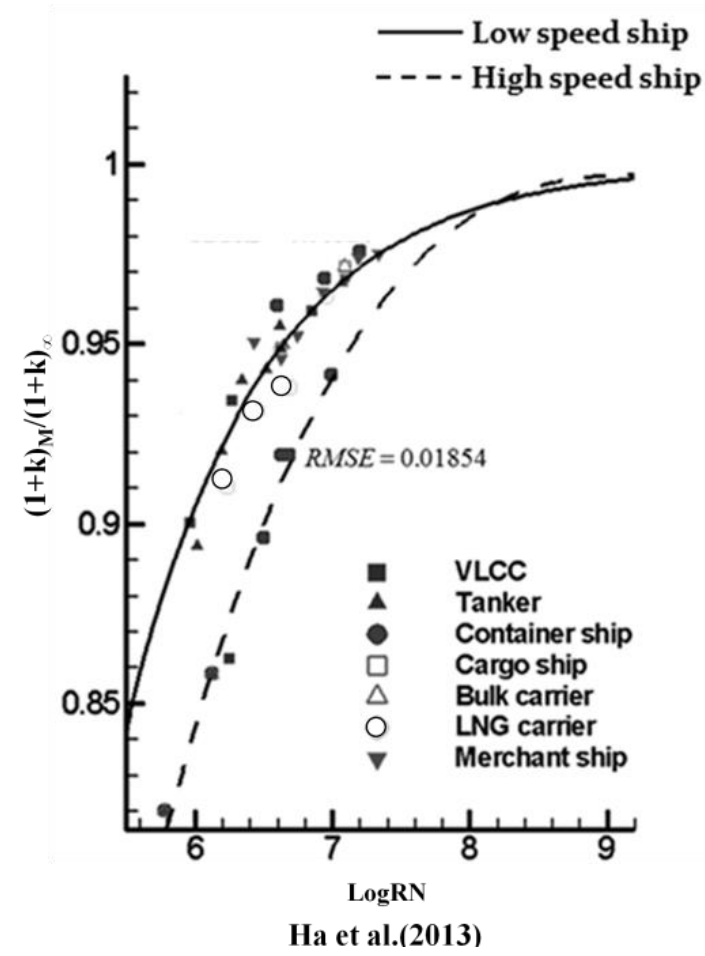

Fig. 12 Comparison of the correlation lines of the form factors

The correlation lines of the form factors were suggested with each middle-low speed ships and high speed ships based on the design speed 20 knots. In this study, the model test data by García-Gómez (2000) and Min and Kang (2010) and the numerical simulation and model test data by this study were used in regression analysis. The regression analysis results by Ha et al. (2013) were applied to the exponential function but only the exponential function applied. In contrast, various functions were applied in the present study. The applied typical functions are Eqs. 1 4 in this study. The hyperbolic function of Eq. 1 was referred in a study reported by Min and Kang (2010).

$$
\begin{aligned}
& \frac{(1+k)_{M}}{(1+k)_{\infty}}=\tanh x, x=a(\log R e) \\
& \frac{(1+k)_{M}}{(1+k)_{\infty}}=a(\log R e)^{n}+b \\
& \frac{(1+k)_{M}}{(1+k)_{\infty}}=a+b \cdot x+c \cdot x^{2} \cdots A x^{n} \\
& \frac{(1+k)_{M}}{(1+k)_{\infty}}=a \cdot e^{b(x+c)}+d
\end{aligned}
$$

Regression analyses were performed. The RMSE (Root Mean Square Error) was smallest in the case of the exponential function. Fig. 13 shows the correlation lines for the form factors, which was constructed from the results of the model test and numerical simulations. The figures show that the correlation line of the high speed vessels such as KCS behaves quite differently from the line for the middle-low speed vessels such as KVLCC2 and KLNG. Therefore, Eq. 5, which is based on an exponential curve and can predict the form factor of a full-scale ship, was used to estimate the correlation lines of the form factors. 


$$
\begin{gathered}
\frac{(1+k)_{M}}{(1+k)_{\infty}}=a \cdot e^{b(x+c)}+d, x=\log \operatorname{Re} \\
a=-1.029, b=-0.828, c=-3.106, d=0.940 \text { for middle-low speed ship } \\
a=-10.780, b=-0.722, c=0.000, d=1.000 \text { for high speed ship }
\end{gathered}
$$

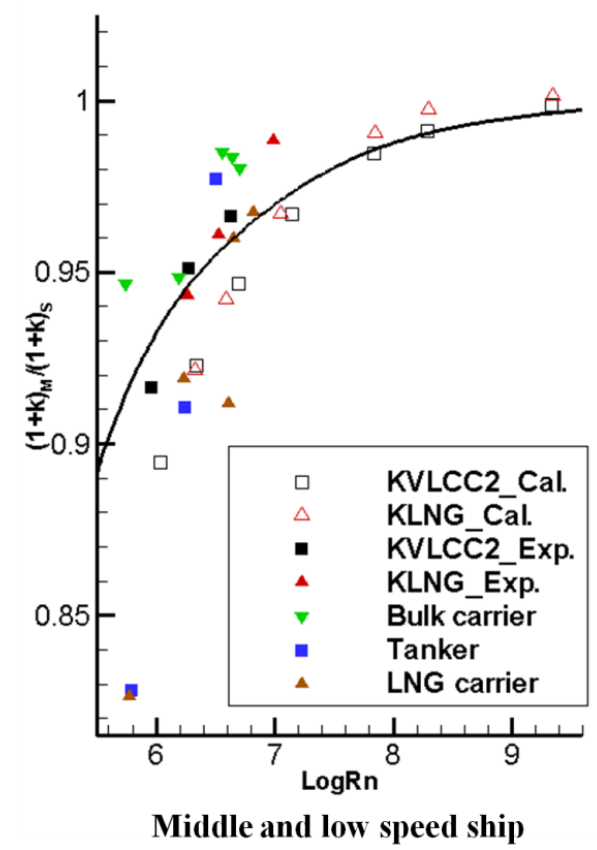

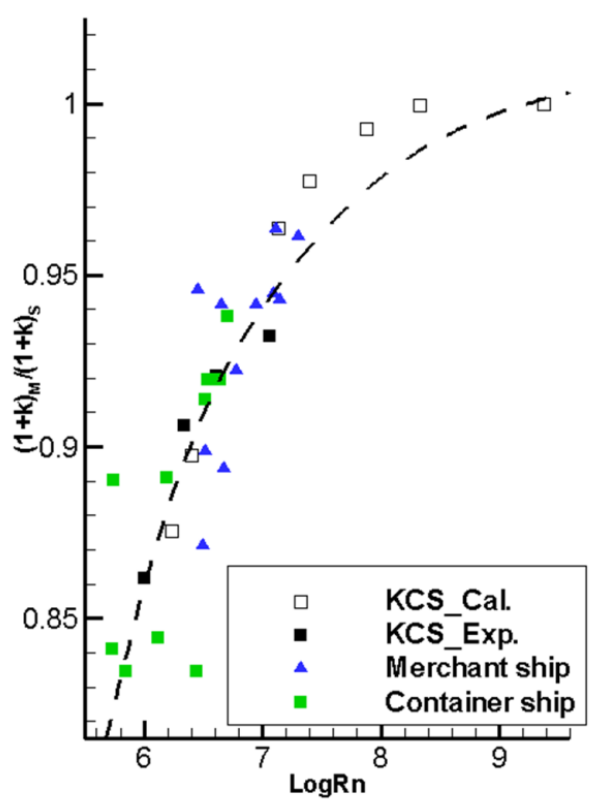

High speed ship

Fig. 13 New correlation lines of the form factors

\subsection{Comparison of the Predicted Results from Four Methods}

The form factors, effective power and delivered power of a full-scale ship was predicted using four different methods, i.e., ITTC 1978 method, Min and Kang (2010), Ha et al., (2013) and the present method. Fig. 14 shows the discrepancy in the result of the predicted effective powers, delivered powers and form factors of full-scale ships using the four different methods. Compared to the form factors of the full-scale using the four different methods, the estimated form factors of the full-scale ships by ITTC' 1978 method differed according to the difference in the Reynolds number due to the model ship size. The estimated form factors of the full-scale ships by Ha et al. (2013) differed according to the difference in the Reynolds number with the model ship sizes. On the other hand, the estimated form factors of the fullscale ships by Min and Kang (2010) and the present study were similar regardless of the Reynolds number according to the model ship size. On the other hand, the predicted effective power of the full-scale ships by Min and Kang (2010) were most different because the correlation line of Min and Kang (2010) was not classified as difference in the hull forms. The predicted the effective power of the full-scale ships by this study were most similar because the correlation line of this study were classified as the differences in the hull forms used the numerical simulation results of the various Reynolds numbers from the model ship sizes to the full-scale ship. In the method reported by Ha et al. (2013), the estimated effective 
powers were more similar than the results estimated by the ITTC' 1978 method and Min and Kang (2010). The effective wake of a full-scale ship is needed to predict the delivered power. The prediction method of the effective wake of a full-scale ship is expressed in Eq. 6, and the form factor of the model ship and full-scale ship were applied to predict the effective wake of a full-scale ship.

$$
W_{S}=\left(t+W_{R}\right)+\left(W_{M}-t-W_{R}\right) \frac{\left(1+k_{S}\right) C_{F S}+\Delta C_{F}}{\left(1+k_{M}\right) C_{F M}}
$$

where, $\mathrm{W}_{\mathrm{R}}$ means a rudder effect. The same form factors of a model ship and full-scale ship were applied to predict the effective wake of a full-scale ship using the ITTC' 1978 method. On the other hand, the axial velocity contours differed according to the model ship sizes in Fig. 4. So the different form factors of a model ship and full-scale ship would be applied to predict the effective wake of a full-scale ship. In other words, the difference in form factors by the Reynolds number were considered for the prediction of the delivered power. The predicted delivered power of the full-scale ship by the ITTC' 1978 method was significantly different with KCS, and the predicted delivered powers of the full-scale ships by Min and Kang (2010) were the most different. The differences in the hull forms were not classified. In KVLCC2, the results of the predicted delivered power of the full-scale ship by Ha et al. (2013) are more different than that by the ITTC' 1978 method. On the other hand, in $\mathrm{KCS}$, the results of the predicted delivered power of the full-scale ship by Ha et al. (2013) were more similar than that by the ITTC' 1978 method. In this study, the results of the predicted delivered powers of the full-scale ships were most similar because this study used the numerical simulation results of the various Reynolds number from the model ship sizes to the full-scale ship, and this study was classified according to the differences in the hull forms. In other words, the correlation lines of this study were proper for the form factor estimation of a full-scale ship. Consequently, the maximum discrepancies in the predicted effective power and delivered power of the full-scale ships occurring due to the changes in the model sizes appeared to be less than approximately $3 \%$, which is far lower than those estimated using existing methods. In particular, this study was more advantageous for the power prediction of high speed hull forms, such as KCS, than the existing methods. Table 3 summarizes the maximum discrepancy in the results due to the variation of the model size. The figure and table show that, for all three test ships, the present method yielded the best results; the discrepancies in the predicted effective powers were confined. 
A Study on the Estimation Method of the form factor for a full-scale ship
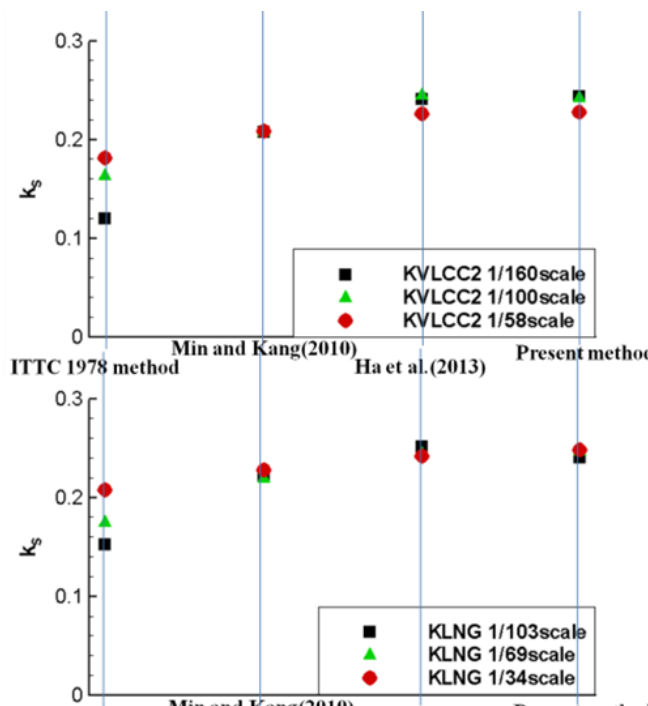

ITTC 1978 method ${ }_{\text {Ha et al.(2013) }}^{\text {Present method }}$
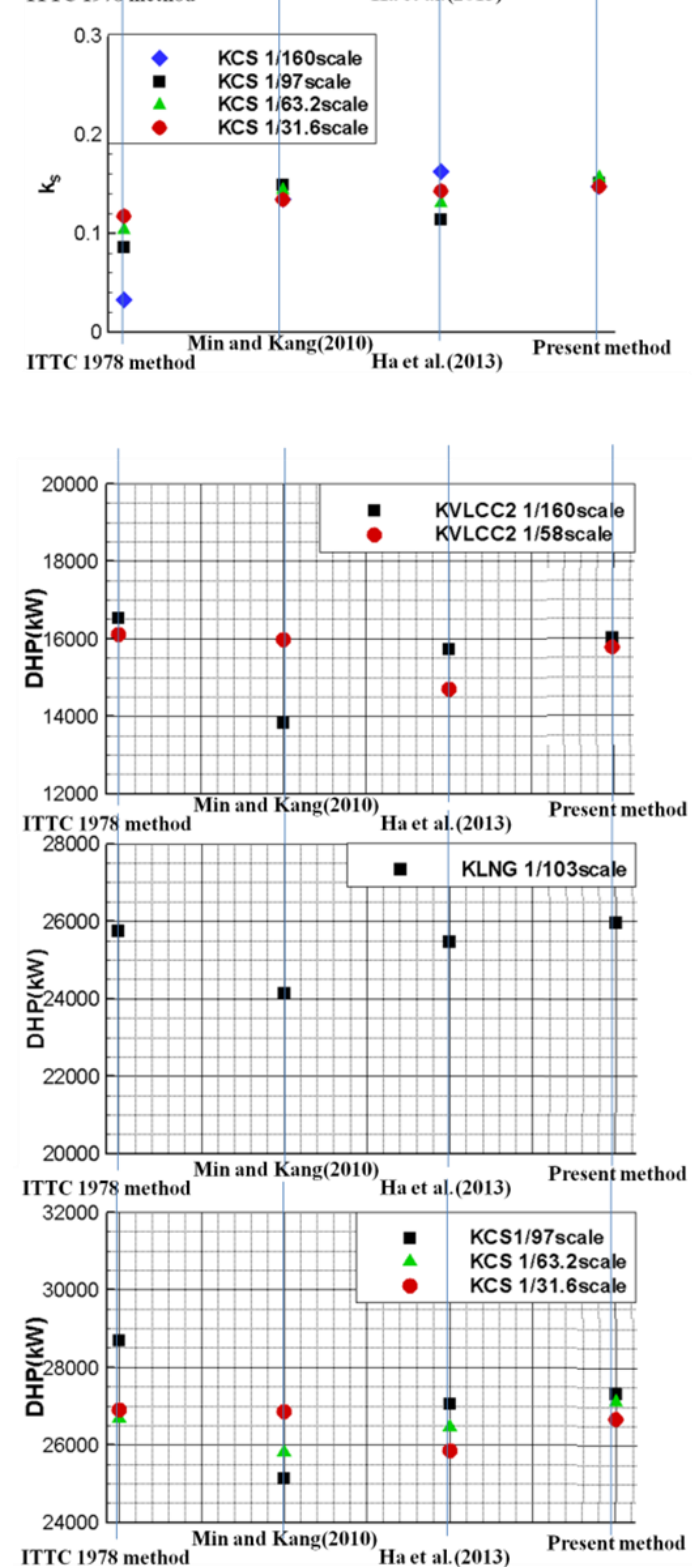

Fig. 14 Comparisons of the form factors, effective powers and delivered powers of the full-scale ships predicted by four different methods
Young-Gill Lee, Yoon-Jin Ha Seunng-Hee Lee, Sang Hyun Kim

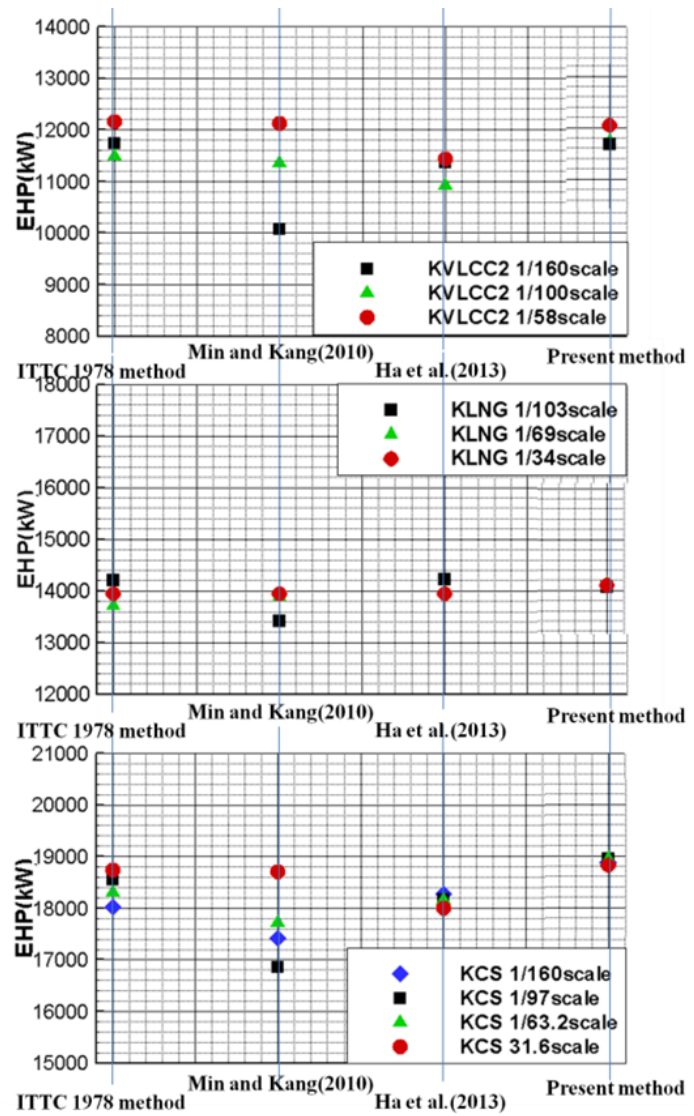


Table 3 Comparisons of the maximum discrepancy of the form factor, effective power and delivered power of the full-scale ships the among four different methods

\begin{tabular}{|c|c|c|c|}
\hline & \multicolumn{3}{|c|}{ KVLCC2 } \\
\hline & $\mathrm{k}$ & EHP & DHP \\
\hline ITTC & 33.7 & 4.5 & 3.6 \\
\hline Min and Kang (2010) & 0.5 & 16.9 & 13.4 \\
\hline Ha et al. (2013) & 6.4 & 3.5 & 5.2 \\
\hline Present method & 0.7 & 2.6 & 1.7 \\
\hline & \multicolumn{3}{|c|}{ KLNG } \\
\hline ITTC & $\mathrm{k}$ & EHP & DHP \\
\hline Min and Kang (2010) & 26.6 & 3.4 & - \\
\hline Ha et al. (2013) & 2.9 & 4.0 & - \\
\hline Present method & 4.1 & 2.1 & - \\
\hline & 0.3 & 2.0 & DHP \\
\hline & \multicolumn{3}{|c|}{ KCS } \\
\hline ITTC & $\mathrm{k}$ & EHP & 6.1 \\
\hline Min and Kang (2010) & 76.2 & 3.8 & 6.4 \\
\hline Ha et al. (2013) & 62.3 & 9.8 & 1.9 \\
\hline Present method & 7.0 & 1.5 & 1.3 \\
\hline
\end{tabular}

\section{Conclusions}

The form factor of a ship during model tests and the same value can be used when extrapolating the results to full-scale. On the other hand, previous studies showed that the form factors vary with the Reynolds number and many attempts have been made to remedy the problem.

In the present study, a practical method was proposed to estimate the form factor of a full scale ship taking the difference in hull forms into consideration by numerical simulations. The present method was applied to predict the power three test ships. The maximum discrepancies in the predicted effective power and delivered power of the full-scale ships occurring due to the changes in the model sizes appeared to be less than approximately $3 \%$, which is far lower than those estimated by the existing methods. In particular, the present method is more advantageous for the power prediction of high speed hull forms, such as KCS, than the existing methods. The major reason is that this method reflects the difference in hull forms when the form factor of the full-scale ship is predicted. Another reason is that when the correlation line of the form factors is derived, the exponential function better reflects the ratio of the estimated form factor of a model and full-scale ship than the hyperbolic function suggested by Min and Kang(2010).

\section{Acknowledgements}

This study was supported by the INHA UNIVERSITY Research Grant. 


\section{REFERENCES}

[1] Choi, J. E., Kim, J. H. and Lee, H. G. (2011). "Computational Study of the Scale Effect on Resistance and Propulsion Performance of VLCC," Journal of the Society of Naval Architects of Korea, 48(3), 222-232. https://doi.org/10.3744/SNAK.2011.48.3.222.

[2] Choi, J. K. and Kim, H. T. (2010). "A Study of Using Wall Function for Numerical Analysis of High Reynolds Number Turbulent Flow, "Journal of the Society of Naval Architects of Korea, 47(5), 647-655. https://doi.org/10.3744/SNAK.2010.47.5.647.

[3] García-Gómez, A. (2000) "On the Form Factor Scale Effect," Ocean Engineering, 26, 97-109. https://doi.org/10.1016/S0029-8018(98)00042-0.

[4] Kim, H. T. and Kim, H. T. (2006). "Research of Element Giving Effects on the Numerical Analysis for the LNG Carrier," Journal of the Society of Naval Architects of Korea, 43(6), 656-666. https://doi.org/10.3744/SNAK.2006.43.6.656.

[5] Kim, W. J. Van, D. H. and Kim, D. H. (2001). "Measurement of flows around modern commercial ship models, "Experiments in Fluids, 31, 567-578. https://doi.org/10.1007/s003480100332.

[6] Min, K. S. and Kang, S. H. (2010). "Study on the Form Factor and Full-Scale Ship Resistance Prediction Method, "Journal of Marine Science Technology, 15, 108-118. https://doi.org/10.1007/s00773-009-0077y.

[7] Raven, H. C., Ploeg, V. D., Starke, A. R. "Towards A CFD-Based Prediction of Ship Performance Progress in Predicting Full-Scale Resistance and Scale Effects."

[8] Yang, H. U., Kim, B. N., Yoo, J. H. and Kim, W. J. (2010). "Wake Comparison between Model and Full Scale Ship Using CFD, " Journal of the Society of Naval Architects of Korea, 47(2), 150-162. https://doi.org/10.3744/SNAK.2010.47.2.150.

[9] Ha, Y. J., Lee, Y. G. and Kang, B. H. (2012). "A Study on the Power prediction of a ship and the Resistance Components Characterisrics by the Geosim Model tests, " Proceeding of the Annual Spring Meeting, KTTC, DaeJeon, Korea.

[10] Ha, Y. J., Lee, Y. G. and Kang, B. H. (2013). "A Study on the Estimation of the Form Factor of FullScale Ship by the Experimental Data of Geosim Models, " Journal of the Society of Naval Architects of Korea, 50(5), 291-297. https://doi.org/10.3744/SNAK.2013.50.5.291.

[11] Van, S. H., Ahn, H., Lee, Y. Y., Kim, C., Hwang, S., Kim, J., Kim, K. S. and Park, I. R. (2011). "Resistance Characteristics and Form Factor Evaluation for Geosim Models of KVLCC2 and KCS, " Proceeding of 2nd International Conference on Advanced Model Measurement Technology for EU Maritime Industry, Newcastle upon Tyne, U. K., 282-293.

Submitted: 13.07.2017. Young-Gill Lee, younglee@inha.ac.kr

Accepted: 11.10.2017. Dept. of Naval Architecture and Ocean Engineering, Inha University, Incheon, Republic of Korea

Corresponding author: Yoon-Jin Ha, yj_ha0811@kriso.re.kr

Korea Research Institute of Ship and Ocean Engineering, DaeJeon,

Republic of Korea

Seung-Hee Lee, shlee@inha.ac.kr

Dept. of Naval Architecture and Ocean Engineering, Inha University, Incheon, Republic of Korea

Sang Hyun Kim, kimsh@inha.ac.kr

Dept. of Naval Architecture and Ocean Engineering, Inha University, Incheon, Republic of Korea 